Published in: J. Nucl. Mater. 363-365, 944-948 (2007)

\title{
Reactivity of soft amorphous hydrogenated carbon films in ambient atmosphere
}

\author{
E. Salançon ${ }^{\mathrm{a}}$, T. Dürbeck ${ }^{\mathrm{b}}$, T. Schwarz-Selinger ${ }^{\mathrm{b}}$, and W. Jacob ${ }^{\mathrm{b} *}$ \\ ${ }^{a}$ Marseille University, UMR 6633, campus de St Jérôme, service 241, 13397 Marseille, France \\ ${ }^{b}$ Max-Planck Institut für Plasmaphysik EURATOM Association, Boltzmannstr. 2, D-85748 Garching, \\ Germany
}

\begin{abstract}
Thermally induced decomposition of soft amorphous hydrocarbon films was investigated by thermal effusion spectroscopy. Released species were detected by a sensitive mass spectrometer. Soft films exhibit a desorption maximum at $\mathrm{T} \approx 760 \mathrm{~K}$ while hard films have their maximum at $\mathrm{T} \approx 875 \mathrm{~K}$. We found a significant reactivity of soft layers towards air and in particular towards humidity in the air. After three month exposure to ambient atmosphere, thermal effusion spectra are significantly different. Water and carbon dioxide peaks increase with exposure time whereas the contributions from hydrocarbons decrease.
\end{abstract}

PACS: 82.80.K, 52.40.H, 68.60.D

JNM Keywords: Amorphization and amorphous material, carbon, hydrogen and hydrides, redeposition

PSI-17 Keywords: Amorphous films, co-deposition, hydrocarbons, thermal desorption, annealing

* Corresponding author address: Boltzmannstr. 2, D-85748 Garching, Germany

* Corresponding author e-mail: Wolfgang.Jacob@ipp.mpg.de 


\section{I- Introduction}

In the present design for the fusion experiment ITER, it is envisaged to build parts of the divertor-the strike zone-from CFC (carbon fiber components) material. This will lead to strong plasma-surface interaction of the boundary plasma with carbon surfaces. The result of this interaction is the erosion of the divertor and the emission of carbon and hydrocarbon compounds into the gas phase. Most of these released species will redeposit in relative close proximity to their place of origin and will form diamond-like amorphous hydrogenated carbon (hard a-C:H) with an atomic ratio of hydrogen isotopes to carbon of about 0.4. However, a small fraction of carbon and hydrocarbon species is not redeposited in the divertor and causes deposition of hydrocarbon layers on surfaces not in direct contact with plasma. Many investigations have been devoted to a study of these redeposited layers $[1,2,3,4,5,6,7,8]$, and it seems clear that they are soft hydrocarbon layers with high $\mathrm{H}$ content. A large fraction of high sticking species contributes to their deposition [9]. Significant amounts of tritium will be retained in the redeposited layers on the wall surface during D-T operation, resulting in a potential environmental risk during accidental vacuum loss because the redeposited films are not stable in air. Causey et al. [10] observed that tritium could be completely released from a redeposited carbon layer (very soft film) due to air exposure at $573 \mathrm{~K}$. Fernandez et al. [11] have shown that after air exposure, the amount of preimplanted deuterium that could be desorbed decreases by a factor of two and that water plays an important role in the formation of deuterated compounds. Wang et al. [12] have shown that hard a-C:H films are eroded during air annealing $(700 \mathrm{~K})$ due to oxidation in contrast to vacuum annealing which results in thermal desorption of hydrogen and hydrocarbons at higher temperature (875 K). Maruyama et al. [13] have shown by infrared spectroscopy that soft a-C:D layers oxidize due to exposure to ambient atmosphere. During annealing in air they erode more easily than hard layers $(550 \mathrm{~K})$. In the present work, the oxidation of soft a-C:H films due to air or humid air exposure are studied by thermal effusion spectroscopy.

\section{II- Experimental}

Details about the experiment and sample preparation will be published elsewhere [14]. In short: The investigated soft a-C:H films with $\mathrm{H} / \mathrm{C}$ ratios of about 0.8 are deposited on silicon substrates by plasma chemical vapor deposition (PCVD) in a capacitively coupled rf glowdischarge using $\mathrm{CH}_{4}$ as feed gas. Prior to deposition the silicon samples are cleaned using oxygen and then hydrogen plasmas. Details of this pre-treatment are given in reference [15]. Pressure during plasma deposition is $2 \mathrm{~Pa}$. To produce soft a-C:H layers, samples are mounted to the grounded electrode which has a distance of $10 \mathrm{~cm}$ opposite to the driven-electrode. The ion energy during deposition on the grounded electrode is estimated to be well below $30 \mathrm{eV}$ so that soft, polymer-like a-C:H films grow [16].

Five samples are presented here; their thickness and optical properties as measured by ex situ ellipsometry at $632 \mathrm{~nm}$ are given in table 1. Ellipsometry measurements were made directly after deposition using an ex-situ ellipsometer. The measured optical properties are typical of soft a-C:H layers. Each sample used for thermal effusion measurements was at least for a short time exposed to ambient atmosphere because the samples had to be transported from the plasma deposition chamber to the thermal effusion experiment. The three samples A1, A2, and A3 are from the same deposition run. They were stored in ambient atmosphere. Some samples were stored after deposition in dry air in a desiccator partially filled with silica gel to remove humidity from the atmosphere (sample B). The shortest time of contact to air was about 10 min (sample C). 
Thermal effusion spectra are measured in the experimental device TESS (Thermal Effusion Spectroscopy Set-up) specifically designed to allow sensitive thermal effusion studies of thin films and surface layers. TESS is an ultrahigh vacuum (UHV) experiment equipped with a Cryopump to provide high pumping speed and a sensitive quadrupole mass spectrometer (QMS). The base pressure of TESS is in the $10^{-9} \mathrm{~Pa}$ range. The QMS is a Pfeiffer/Inficon DMM 422 equipped with a cross-beam electron source. The electron energy in the ion source of the QMS was set to $40 \mathrm{eV}$ for all experiments and the emission current was set to $0.6 \mathrm{~mA}$. The temperature of the sample holder is measured by a Chromel/Alumel $(\mathrm{Ni} / \mathrm{NiCr})$ thermocouple. It allows generating a linear temperature ramp controlled by a PID controller (Eurotherm 902P). The temperature ramps used in the five experiments presented here are $15 \mathrm{~K} / \mathrm{min}$ or $30 \mathrm{~K} / \mathrm{min}$. We have checked that the two temperature ramps yield identical thermal effusion spectra of samples presented here so that we can compare the normalized shapes.

\section{III- Results and discussion}

Thermal effusion spectra (TE spectra) for the five investigated samples are presented in Fig. 1. The normalized mass spectrometry signal for $15 \mathrm{amu}$ is shown. The $16 \mathrm{amu}$ signal yields similar spectra, but in some cases water contributes to it. We assign the $15 \mathrm{amu}$ signal to the $\mathrm{CH}_{3}$ ion which is produced by dissociative ionization from methane and higher hydrocarbons in the ionizer of the QMS. The spectrum of sample $C$ shows two distinct maxima at 760 and $820 \mathrm{~K}$. Sample B shows a similar shape as C, but the intensity ratio of peaks 1 and 2 changes significantly. A1 shows a very similar spectrum as B while for A2 and A3 the first peak has almost completely disappeared. Sample $C$ had the shortest contact to air (about $10 \mathrm{~min}$ ). Sample B was exposed to air for about $15 \mathrm{~min}$ and stored for 68 days in a desiccator in dry air. It shows a very similar spectrum as sample A1 which was exposed to air for about 3 days. Samples A2 and A3 were stored in air for 34 and 95 days, respectively. In our TE spectra we are usually recording 29 mass channels in the range from 1 to $95 \mathrm{amu}$. Most other measured mass channels exhibit only one desorption peak with a peak temperature around $760 \mathrm{~K}$. We note that all mass channels other than 15 and 16 amu show no corresponding change of peak shape but a change of intensity.

From the comparison of samples A1, A2, and A3, which were deposited in the identical deposition run, we can clearly recognize the influence of storage in air. It seems that the reaction of this layer is almost complete after 34 days. In any case, the difference between the spectra of samples A2 and A3 (34 days vs. 95 days) are rather small. Sample B which was stored for 68 days in dry air shows a spectrum very similar to A1. We, therefore, conclude that the reaction was either completely suppressed or proceeded much slower in that case. The sample with the shortest contact to air, sample C, shows a very strong first peak and only a weak second peak. From this sequence of TE spectra we speculate that the peak at $820 \mathrm{~K}$ occurs only if the sample was exposed to air. Unfortunately, the present experimental set-up does not allow to measure TE spectra without any intermediate contact to air.

We further hypothesize that the water of the ambient air is responsible for the observed changes of the layers. Sample B which was stored for 68 days in dry air has a spectrum comparable to that of A1, but for sample A2, which was for 34 days in ambient air, the first peak has almost completely disappeared. This suggests that water is the most important reactant. To test this hypothesis we stored an additional sample for three months in humid air. This sample was placed in a box with two compartments, one containing water and the other our sample. A picture of this sample is shown in Fig. 2. The appearance of the sample changed significantly. The surface is covered with stains (large grey areas in Fig.2) and little holes (small dots in the black areas). Inspections with a microscope revealed that the large 
grey stains are areas which are riddled with a network of cracks. It appears that in these areas the layer has disintegrated into little chips which still adhere to the silicon substrate. Identical samples stored for the same time in normal air are still intact and uniform, even after 10 months. From this experimental observation we draw the conclusion that a chemical reaction took place between the soft a-C:H layer and water from the air.

In Fig. 3 we present a quantitative comparison of TE spectra of a number of mass channels to demonstrate the difference between samples A1 and A3 (3 days vs. 95 days in ambient air). Since a quantitative comparison of mass spectrometry signals over long time scales is not a priori guaranteed, we measured in addition samples from the same deposition run which were stored in vacuum. The TE spectrum for an identical sample as A1 stored for three month in vacuum is identical to the spectrum of sample A1. So, we are very confident that we can make this quantitative comparison of our mass spectrometry signals. For the mass $2 \mathrm{amu}\left(\mathrm{H}_{2}\right)$ signal, the shapes of the thermal effusion spectra are similar, but the intensity for A1 is much higher than for A3. This phenomenon, particularly well visible for $2 \mathrm{amu}$, is observed for all mass channels except for those which are described in the following paragraph. In addition, the total pressure rises during the TE experiments of A1 and A3 show similar peak shapes as the 2 amu curves in Fig. 3. The maximum for A3 is about 30\% lower than for A1 and slightly shifted to higher temperature.

For mass 15 the peak shape is changing as described in Fig. 1. The peak integral of A3 is slightly lower than that of A1. Masses 18 and 44 keep their shape, but the intensities from A1 are smaller than from A3. Mass 17, not plotted in Fig. 3, shows the same behavior as mass 18. Only the three masses 17, 18, and 44 exhibit a converse behavior compared with all other mass peaks and the total pressure rise. This indicates that the formed reaction products contribute to 17, 18 and $44 \mathrm{amu}$. These mass peaks correspond most probably to water and carbon dioxide. This interpretation is corroborated by published data. Maruyama et al. [13] have shown by infrared spectroscopy that chemically bonded $\mathrm{OH}, \mathrm{CO}$, and other $\mathrm{COH}$ groups are formed in soft layers by reaction with air. They can be considered as precursors for volatile $\mathrm{H}_{2} \mathrm{O}$, $\mathrm{CO}$ and $\mathrm{CO}_{2}$ species forming during TE experiments. Of course, we also anticipate formation of $\mathrm{CO}$ as decomposition product. But the integral of mass 28 for A3 is lower than that for A1. In this case, the interpretation of the mass spectrometric results is complicated by the fact that other species, in particular $\mathrm{C}_{2} \mathrm{H}_{\mathrm{x}}(\mathrm{x}=4,6)$ and higher hydrocarbons, contribute to mass 28. Obviously, the reduced contribution from hydrocarbons to the 28 amu peak overcompensates the increased contribution from CO.

The influence of water was already pointed out by Fernandez et al. [11] with an increase of the HDO signals during thermal effusion of graphite implanted by deuterium plasma and exposed to atmospheric air. They conclude that this HDO comes from incorporation of $\mathrm{OH}$ groups. Maruyama et al. [13] have shown that deuterium in soft a-C:D layers is replaced by oxygen, hydrogen, and most probably also $\mathrm{OH}$ groups if the samples are annealed at moderate temperatures in ambient atmosphere. They concluded that deuterium was lost either as $\mathrm{D}_{2}$ or through the formation of deuterated water, $\mathrm{D}_{2} \mathrm{O}$ or HDO. For the erosion process (due to annealing in air) they propose as the final step the formation of volatile oxides $\left(\mathrm{CO}\right.$ and $\left.\mathrm{CO}_{2}\right)$ which leads to the removal of carbon. We have shown here that precursors for the formation of water and $\mathrm{CO}_{2}$ in soft a:C-H films appear in ambient atmosphere already at room temperature. This reaction is initially very fast (timescale of minutes); we assume that it occurs as soon as the sample is exposed to air, mainly due to reaction of water with the sample. The initially fast reaction slows down considerably and further changes occur on a timescale of days to weeks. 


\section{IV- Conclusions}

Soft, polymer-like hydrocarbon layers, which represent a model system to study the behavior of redeposited layers in remote areas of fusion devices, were investigated by thermal effusion spectroscopy. We found that these soft layers react on a timescale of minutes to days if exposed to ambient atmosphere. This reaction causes a significant change of the thermal effusion spectrum of the mass 15 and 16 amu peaks, a significant reduction of the $\mathrm{H}_{2}$ signal, and an increase of the signals of water and $\mathrm{CO}_{2}$. The results indicate that mostly water in the ambient atmosphere is responsible for this reaction.

This reactivity of soft a-C:H layers towards ambient atmosphere must always be kept in mind if redeposited layers from fusion experiments are to be investigated. Even short exposure to air can change the chemistry of the layers significantly. Important consequences of air contact are the incorporation of oxygen and the loss and exchange of hydrogen. Further studies investigating the oxygen uptake and hydrogen isotope exchange using a-C:D layers are underway.

\section{Tables}

\begin{tabular}{ccc|c|c|c}
\hline & $\mathbf{n}(632 \mathrm{~nm})$ & $\mathrm{k}$ & thickness & Exposure time & Temperature ramp \\
\hline $\mathrm{A} 1$ & 1.56 & 0.001 & $400 \mathrm{~nm}$ & 3 days : air & $15 \mathrm{~K} / \mathbf{m i n}$ \\
\hline $\mathrm{A} 2$ & & & & 34 days : air & $15 \mathrm{~K} / \mathbf{m i n}$ \\
\hline $\mathrm{A} 3$ & & & 95 days : air & $15 \mathrm{~K} / \mathbf{m i n}$ \\
\hline $\mathrm{B}$ & 1.58 & 0.001 & $220 \mathrm{~nm}$ & $\begin{array}{c}10 \mathrm{~min} \text { air } \\
+68 \text { days: dry air }\end{array}$ & $30 \mathrm{~K} / \mathbf{m i n}$ \\
\hline $\mathrm{C}$ & 1.57 & 0.001 & $400 \mathrm{~nm}$ & $10 \mathrm{~min}($ air $)$ & $30 \mathrm{~K} / \mathbf{m i n}$ \\
\hline
\end{tabular}

Table 1: Optical properties and thickness of the investigated samples as determined by ex-situ ellipsometry. Also given are the exposure conditions and the temperature ramps for the thermal effusion investigations. 


\section{Figures}

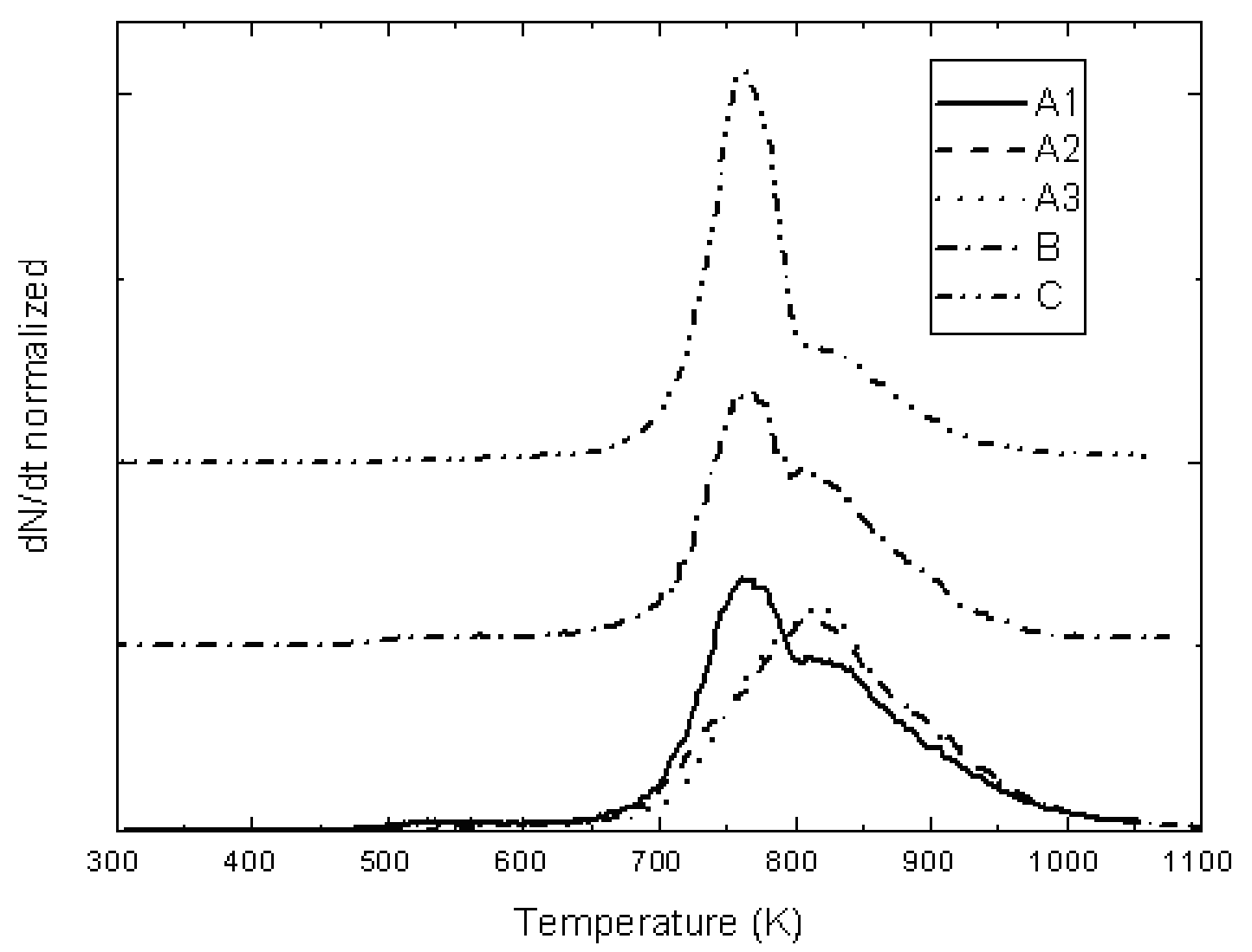

Figure 1: Normalized QMS signal for the mass 15 amu for the 5 different samples described in table 1 . The spectra are normalized to yield the same peak integral.

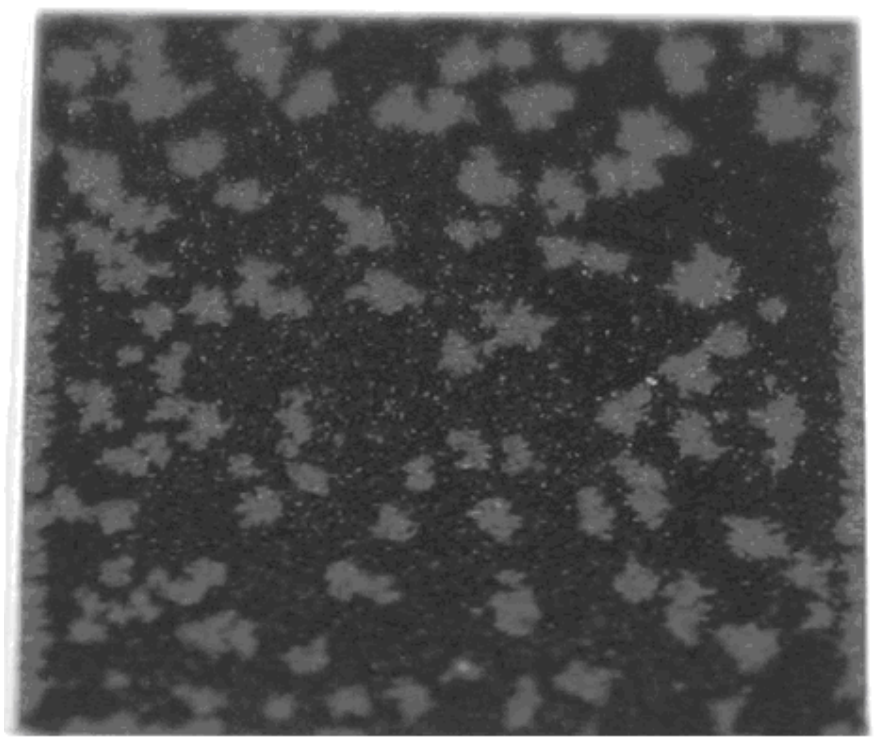

Figure 2: Photo of a soft a-C:H sample stored for three months in humid air (from the same deposition run as samples A). 


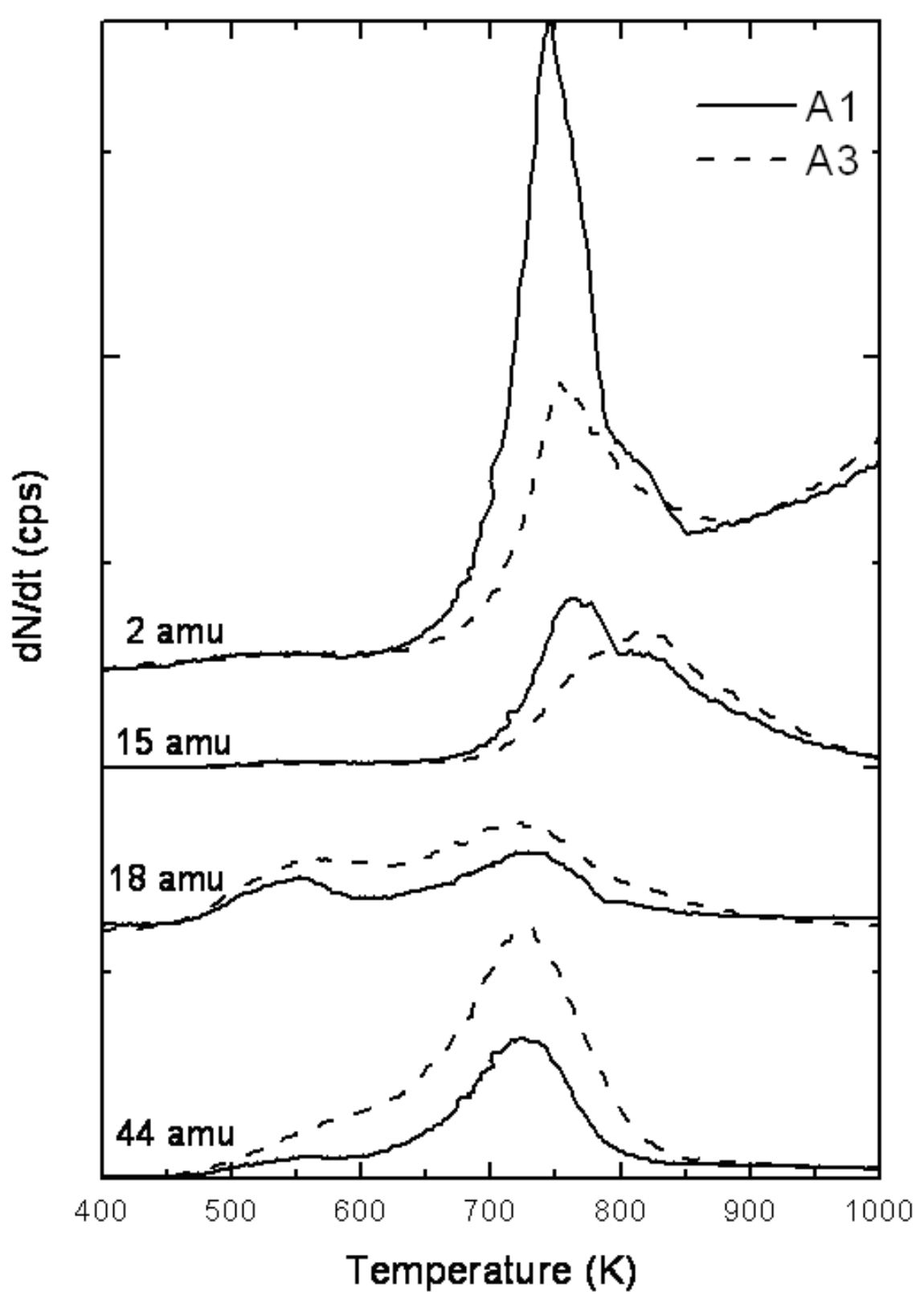

Figure 3: Quantitative comparison of different mass channels of thermal effusion spectra of the two samples A1 and A3. From top to bottom: 2 amu, $15 \mathrm{amu}, 28 \mathrm{amu}$, and $44 \mathrm{amu}$. The firsts two show a decrease of the corresponding signal while the others two show an increase. 


\section{References}

[1] J.P. Coad, N. Bekris, J.D. Elder, S. Erents, D. Hole, K. Lawson, G. Matthews, R. Penzhorn, P. Stangeby, J. Nucl. Mater. 290-293 (2001) 224

[2] M. Rubel, J.P. Goad; N. Bekris, S. Erents, D. Hole, G. Matthews, R. Penzhorn, Contributors to the EFDA-JET work programme, J. Nucl. Mater. 313-316 (2003) 321

[3] M. Mayer, A. von Keudell, V. Rohde, P. Coad, JET-EFDA contributors, in: R. Koch, S. Lebedev (Eds.), Proceedings of the 30th EPS Conference on Controlled Fusion and Plasma Physics, European Physical Society, 2003, European Conference Abstracts Vol. 27A, O-2.6A

[4] C. Hopf, K. Letourneur, W. Jacob, T. Schwarz-Selinger, A. von Keudell, Appl. Phys. Lett. 74 (1999) 3800

[5] C. Hopf, T. Schwarz-Selinger, W. Jacob, A. von Keudell, J. Appl. Phys. 87 (2000) 2719

[6] A. von Keudell, C. Hopf, T. Schwarz-Selinger, W. Jacob, Nucl. Fus. 39 (1999) 1451

[7] M. Mayer, V. Rohde, A. von Keudell, ASDEX Upgrade Team, J. Nucl. Mater. 313-316 (2003) 429

[8] V Rohde, M. Mayer, J. Likonen, R. Neu, T. Puetterich, E. Vainonen-Ahlgren, ASDEX Upgrade Team, J. Nucl. Mater. 337-339 (2005) 847

[9] W. Jacob, J. Nucl. Mater. 337-339 (2005) 839

[10] R.A. Causey, W.L. Chrisman and W.L. Hsu, J. Vac. Sci. Technol. A 7 (1988) 1078

[11] V. Fernandez, J. Bardon, J.P. Palmari, C. Grisolia, J. Nucl. Mat. 176 \& 177 (1990) 1005

[12] W. Wang, W. Jacob, J. Roth, J. Nucl. Mat. 245 (1997) 66-71

[13] K. Maruyama, W. Jacob, J. Roth, J. Nucl. Mater. 264 (1999) 56

[14] E. Salançon, T. Dürbeck, T. Schwarz-Selinger, W. Jacob, to be published

[15] T. Schwarz-Selinger, A. von Keudell, W. Jacob, J. Appl. Phys. 86, (1999) 3988

[16] W. Jacob, Thin Solid Films 326 (1998) 1 